\title{
Identification of blood-derived candidate gene markers and a new 7-gene diagnostic model for multiple sclerosis
}

\author{
Xin Chen, Huiqing Hou, Huimin Qiao, Haolong Fan, Tianyi Zhao and Mei Dong ${ }^{*}$
}

\begin{abstract}
Background: Multiple sclerosis (MS) is a central nervous system disease with a high disability rate. Modern molecular biology techniques have identified a number of key genes and diagnostic markers to MS, but the etiology and pathogenesis of MS remain unknown.

Results: In this study, the integration of three peripheral blood mononuclear cell (PBMC) microarray datasets and one peripheral blood T cells microarray dataset allowed comprehensive network and pathway analyses of the biological functions of MS-related genes. Differential expression analysis identified 78 significantly aberrantly expressed genes in MS, and further functional enrichment analysis showed that these genes were associated with innate immune response-activating signal transduction $(p=0.0017)$, neutrophil mediated immunity $(p=0.002)$, positive regulation of innate immune response $(p=0.004)$, IL-17 signaling pathway $(p<0.035)$ and other immune-related signaling pathways. In addition, a network of MS-specific protein-protein interactions (PPI) was constructed based on differential genes. Subsequent analysis of network topology properties identified the up-regulated CXCR4, ITGAM, ACTB, RHOA, RPS27A, UBA52, and RPL8 genes as the hub genes of the network, and they were also potential biomarkers of MS through Rap1 signaling pathway or leukocyte transendothelial migration. RT-qPCR results demonstrated that CXCR4 was obviously up-regulated, while ACTB, RHOA, and ITGAM were down-regulated in MS patient PBMC in comparison with normal samples. Finally, support vector machine was employed to establish a diagnostic model of MS with a high prediction performance in internal and external datasets (mean AUC $=0.97$ ) and in different chip platform datasets $(\mathrm{AUC}=(0.93)$.
\end{abstract}

Conclusion: This study provides new understanding for the etiology/pathogenesis of MS, facilitating an early identification and prediction of MS.

Keywords: Biomarker, Support vector machine approach, Multiple sclerosis, Bioinformatics, Protein-protein interaction

\section{Background}

Multiple sclerosis (MS), which is a chronic inflammatory disease at the central nervous system of autoimmune etiology [1], is characterized by varying degrees of demyelination and axonal loss. MS predominantly affects young

*Correspondence: dm-mp@126.com

Department of Neurology, The Second Hospital of Hebei Medical

University, Shijiazhuang 050000, Hebei, China women (between the ages of 20 and 40) and is a leading cause of disability among young adults in the United States [2]. About 2.5 million cases have been reported all over the world, with about 400,000 taking place in the United States, moreover, the number of cases is expected to increase in the future [3-5]. Multiple factors including Epstein-Barr virus (EBV) infection [6], Vitamin D deficiency [7], smoking [8], and a high sodium diet [9] all contribute to the risk of developing MS. The pathogenesis

(c) The Author(s) 2021. This article is licensed under a Creative Commons Attribution 4.0 International License, which permits use, sharing, adaptation, distribution and reproduction in any medium or format, as long as you give appropriate credit to the original author(s) and the source, provide a link to the Creative Commons licence, and indicate if changes were made. The images or other third party material in this article are included in the article's Creative Commons licence, unless indicated otherwise in a credit line to the material. If material is not included in the article's Creative Commons licence and your intended use is not permitted by statutory regulation or exceeds the permitted use, you will need to obtain permission directly from the copyright holder. To view a copy of this licence, visit http://creativeco mmons.org/licenses/by/4.0/. The Creative Commons Public Domain Dedication waiver (http://creativecommons.org/publicdomain/ zero/1.0/) applies to the data made available in this article, unless otherwise stated in a credit line to the data. 
of MS involves an immune attack against central nervous system (CNS) antigens, resulting in a sustained autoreactive T-cell Peripheral activation $[10,11]$, Then postactivation myelin-reactive $T$ Cells are able to penetrate the blood-brain barrier (BBB) into the central nervous system [12] to recruit other inflammatory cells, including $\mathrm{T}$ cells, monocytes, and B cells[13]. Long-term activation of microglia and macrophages will lead to destruction of myelin [14], and the activate resident glial cells such as microglia will cause persistent inflammation [15]. So far, however, immunomodulator therapy and symptomatic treatment are considered as two main strategies for treating MS, but they can only improve the body functions [16]. Several studies have shown that susceptibility to MS is genetically dependent $[17,18]$, but the specific genetic factors remain largely unknown. Therefore, the identification of risk alleles or candidate genes that play important role in the pathogenesis of MS remains a challenge.

MS, which is a chronic, progressive, immune-mediated disorder of the CNS, is characterized by neurodegeneration resulted from inflammation, demyelination and Axonal damage [19]. Currently, we still lack early diagnosis and management of MS [20], thus, biomarkers effective for MS identification are urgently needed. Bioinformatics analysis of gene expression profiles facilitate the screening of MS biomarkers. Dorothee Nickles et al. [21] compared the gene expression profiles of wholeblood RNA samples derived from both healthy individuals and MS patients, and identified abnormal individual transcripts and biological pathways in MS patients. Jeffrey $M$ Trent et al. [22] performed gene expression profiling on peripheral blood mononuclear cells for identifying MS-related candidate genes by cDNA microarrays; Teresa Maria Creanza et al. [23] adopted differential network approach and demonstrated that MS networks showed a low connectivity relative to health status, and they also proved that interferon treatment can activate gene transcription. These studies have demonstrated the feasibility of screening key biomarkers for MS based on gene expression profiles. They designed different analysis strategies and got different results. It is well known that there are multiple optimal solutions for high-dimensional data analysis, but it is worth mentioning that these studies have focused on a single dataset of MS patients, in comparison, gene expression profiling of large samples from multiple cohorts to identify biomarkers will be more reliable in developing new biomarkers for early prevention and management of MS.

Biomarkers for MS can help diagnose, predict the disease course or determine the outcome of treatment response. Although biomarkers and extensive research are needed to identify them, the validation and clinical application of biomarkers in multiple sclerosis remains unmet. There is still a large gap between exploratory biomarkers proposed in many studies, proven biomarkers, and biomarkers incorporated into routine clinical practice.

This study used high-throughput gene expression profiles from a large cohort of MS patients to investigate the alterations of expression profiling patterns between MS patients and healthy individuals, aiming to identify potential biomarkers and to develop a diagnostic model of MS patients.

\section{Results}

Identification of differentially expressed gene between MS samples and healthy controls samples

Data sets GSE21942, GSE43591 and GSE17048 were obtained from GEO and standardized for consistent data distribution (Fig. 1a). 78 differentially expressed gene (DEGs) were finally screened after data pre-processing and quality control. Among the 78 DEGs, 47 genes were down-regulated in the disease group, while 31 genes were down-regulated in the healthy group, and the fold change heatmap of these DEGs in each dataset is shown in Fig. 1b. The GSEA enrichment results of the DEGs in each data set are shown in Fig. 1c, and it can be seen that DEGs were mainly enriched in the group with high fold change in each data set.

\section{Functional enrichment analysis of DEGs}

To better understand the functional involvement of the DEGs, GO and KEGG functional enrichment analysis were performed on the 78 DEGs. The results showed 186 enriched GO terms, which were mainly enriched to toll-like receptor signaling pathway, pattern recognition receptor signaling pathway, innate immune response-activating signal transduction (Fig. 2a). Interestingly, these enriched pathways are important biological pathways involved in the immune process, and this is consistent with the nature of MS as an immune system disease. In addition, these genes were also enriched to bacterial invasion of epithelial cells, leukocyte transendothelial migration, chemokine signaling pathway, regulation of actin cytoskeleton, yersinia infection, ribosome, and IL-17 signaling pathway (Fig. 2b). Several of above listed pathways are associated with the development of MS, such as the IL-17 signaling pathway, which plays an important role in the pathogenesis of many autoimmune diseases including MS [24], and leukocyte transendothelial migration, which is a key feature of MS pathology [25]. 


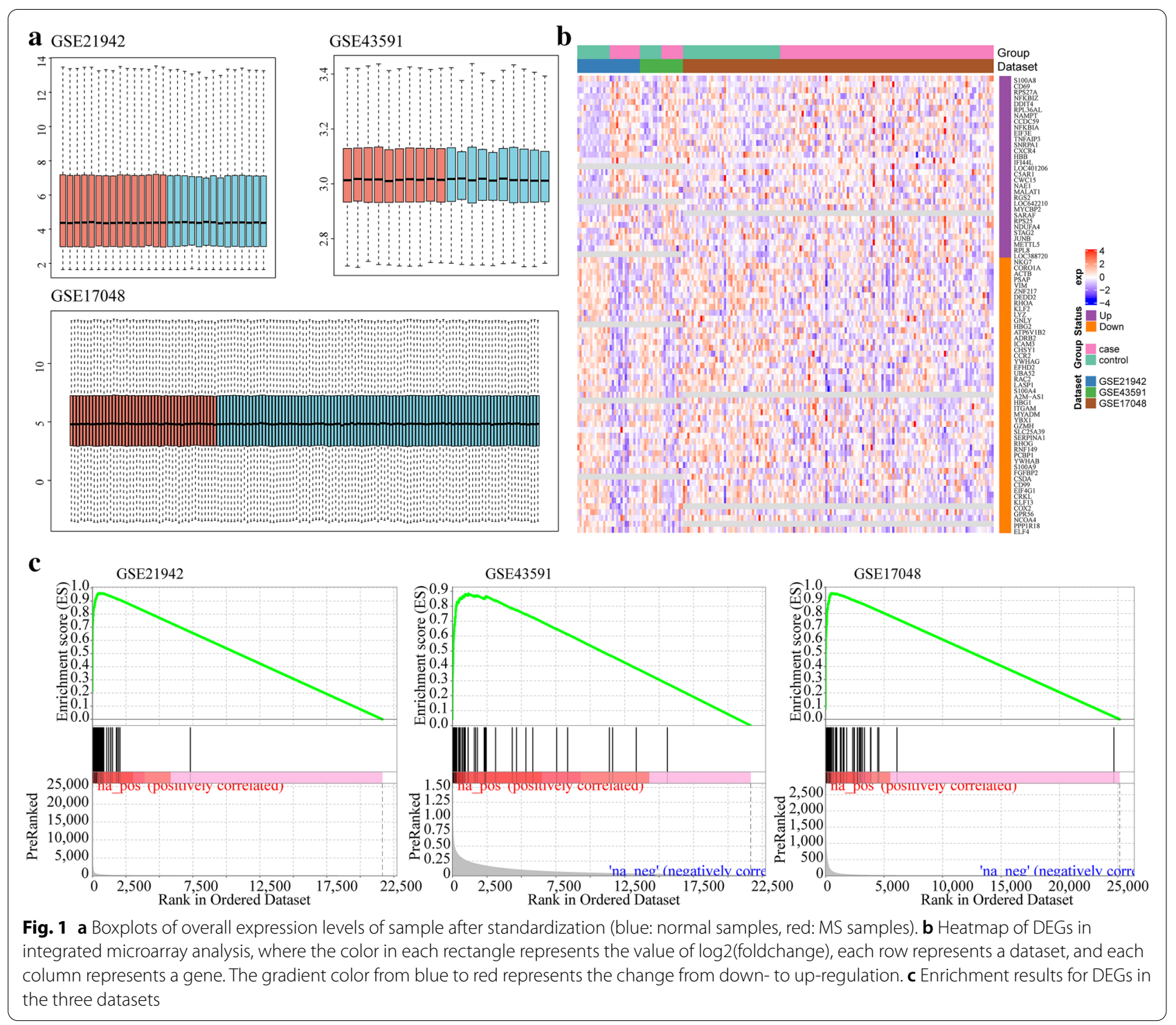

\section{Identification of potential biomarkers associated with MS diagnosis}

To examine the interactions among the 78 DEGs, each gene was mapped into the String database to obtain gene interactions, according to the threshold of a minimum required interaction score $>0.4$ (medium confidence). After visualizing the gene interactions by Cytoscope, we found that the 78 DEGs were mapped into a total of 124 interactions in the network (Fig. 3a). The cytoHubba plugin in Cytoscope was used for hub gene identification, with the employment of three calculation methods (Degree, Closeness and Betweenness). The sub-networks of the top 10 genes evaluated by the three calculation methods are shown in Fig. 3b-d. It can be seen that hub genes with high degree, closeness and betweenness were generally consistent and showed interaction with multiple genes in the network. We further analyzed the topological properties of the network, and found that the distribution of degrees in the network (Fig. 3e) demonstrated a power-law distribution, which is consistent with biological network characteristics, as most of the gene degrees were less than 7. In addition, by calculating the closeness of the network, we found that most of the nodes have an overall high closeness of basically above 15 (Fig. 3f). Finally, the betweenness of the network was calculated and most nodes had a betweenness below 100 (Fig. 3g). Nodes with high degree, closeness or betweenness were considered as important nodes in the network. Top $5 \%, 10 \%, 15 \%, 20 \%, 25 \%, 30 \%$ of the nodes with the highest degree, Closeness, and Betweenness as hub genes 


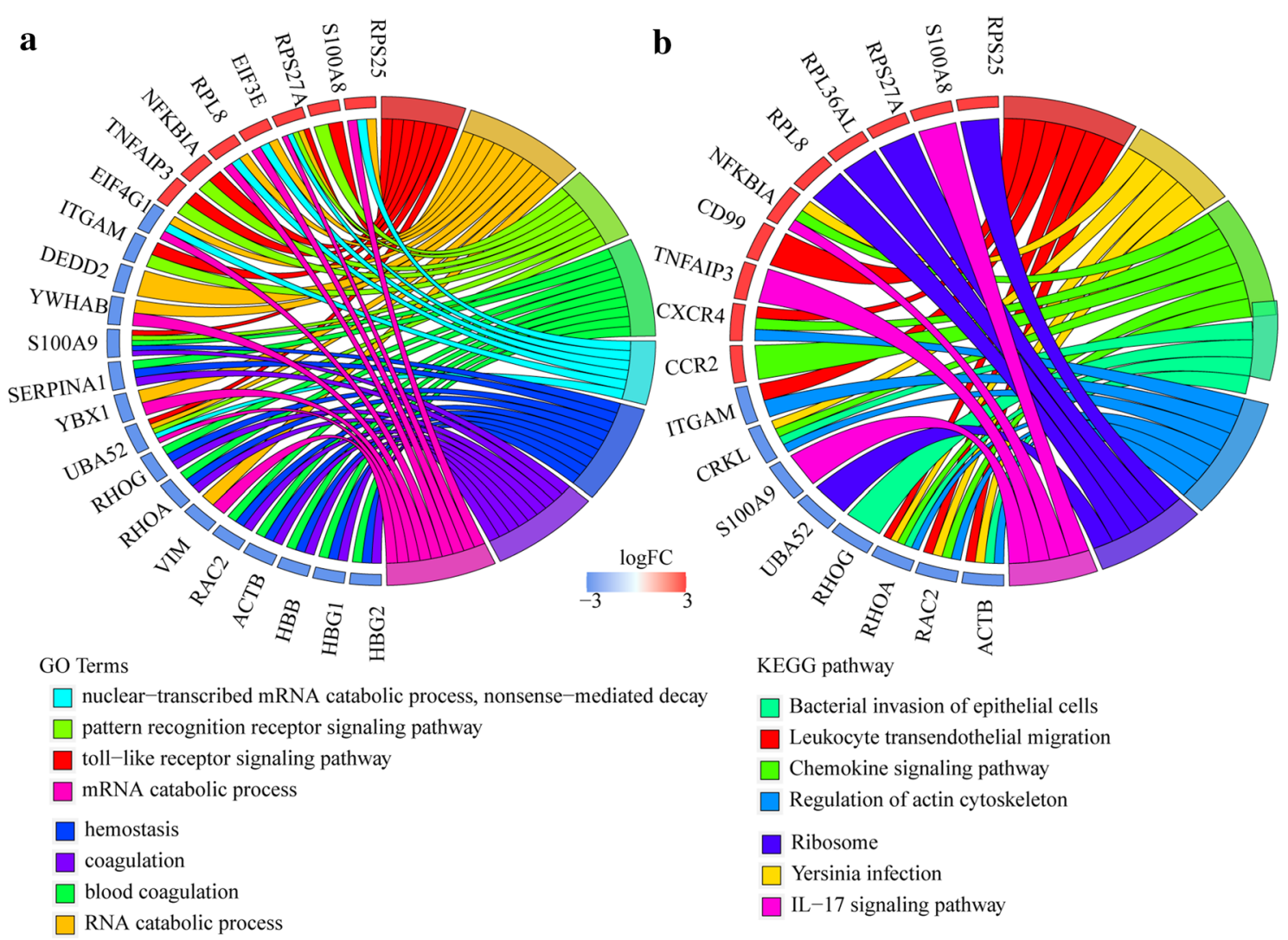

Fig. 2 Functional enrichment analysis of 78 DEGs. a Enriched GO terms of DEGs. b Enriched KEGG biological pathways of DEGs. Different colors represent different functions, and the hyphen represents gene correspondence enrichment to GO term or KEGG biological pathways

of the network were selected respectively, and GSE17048 was used as the training set to observe the classification performance under different thresholds (Additional file 1: Figure S1A). With the increase of the threshold, the number of included genes gradually increased, and the AUC also gradually increased. When the AUC rose slowly after $20 \%$, we chose the top $20 \%$ as the threshold. By selecting the top $20 \%$ of the nodes with the highest degree, closeness, and betweenness as hub genes of the network, here, seven hub genes, namely, CXCR4, ITGAM, ACTB, RHOA, RPS27A, UBA52, and RPL8, were determined. Specifically, CXCR4 has multifunctional effects, and is widely involved in a variety of pathological conditions, including immune diseases, viral infections and cancer [26]. RhoA, which is a ubiquitously expressed cytoplasmic protein, belongs to the small GTPase family of enzymes, and acts as a molecular switch and is activated in response to the binding of chemokines, cytokines and growth factors. Mutations in Rho and Rho regulatory factors predispose to autoimmune diseases and are the cause of malignancies of the hematopoietic system [27]. These findings suggest that the seven hub genes could serve as potential biomarkers for MS, and that it is effective to mine MS-associated markers by constructing MS-specific protein interaction networks.

\section{Construction and verification of the MS diagnostic model}

We used GSE17048 as a training dataset $(\mathrm{N}=144$, $\mathrm{MS}=99$, Normal $=45), \mathrm{GSE} 21942(\mathrm{~N}=29, \mathrm{MS}=14$, Normal $=15)$ and GSE43591 $(\mathrm{N}=20, \mathrm{MS}=10$, Nor$\mathrm{mal}=10)$ as validation sets, and GSE15245 $(\mathrm{N}=65$, $\mathrm{MS}=51$, Normal $=14$ ) as the independent validation set. Seven hub genes served as features in training data set, and their corresponding gene expression profiles were obtained. Then, the classification model was established by support vector machine (SVM). By applying tenfold cross-validation in the model test, 134 out of the 144 samples were correctly classified, with a classification accuracy of $93.06 \%$, model sensitivity to MS of $96.97 \%$, specificity of $84.44 \%$, and area under the ROC curve (AUC) was 0.907 (Fig. 4a). Furthermore, the established model was used to predict the samples in the validation data set to test the prediction ability of the model. In the validation datasets GSE21942 and GSE43591, all the samples were correctly classified, with a classification accuracy of $100 \%$, moreover, the sensitivity and 


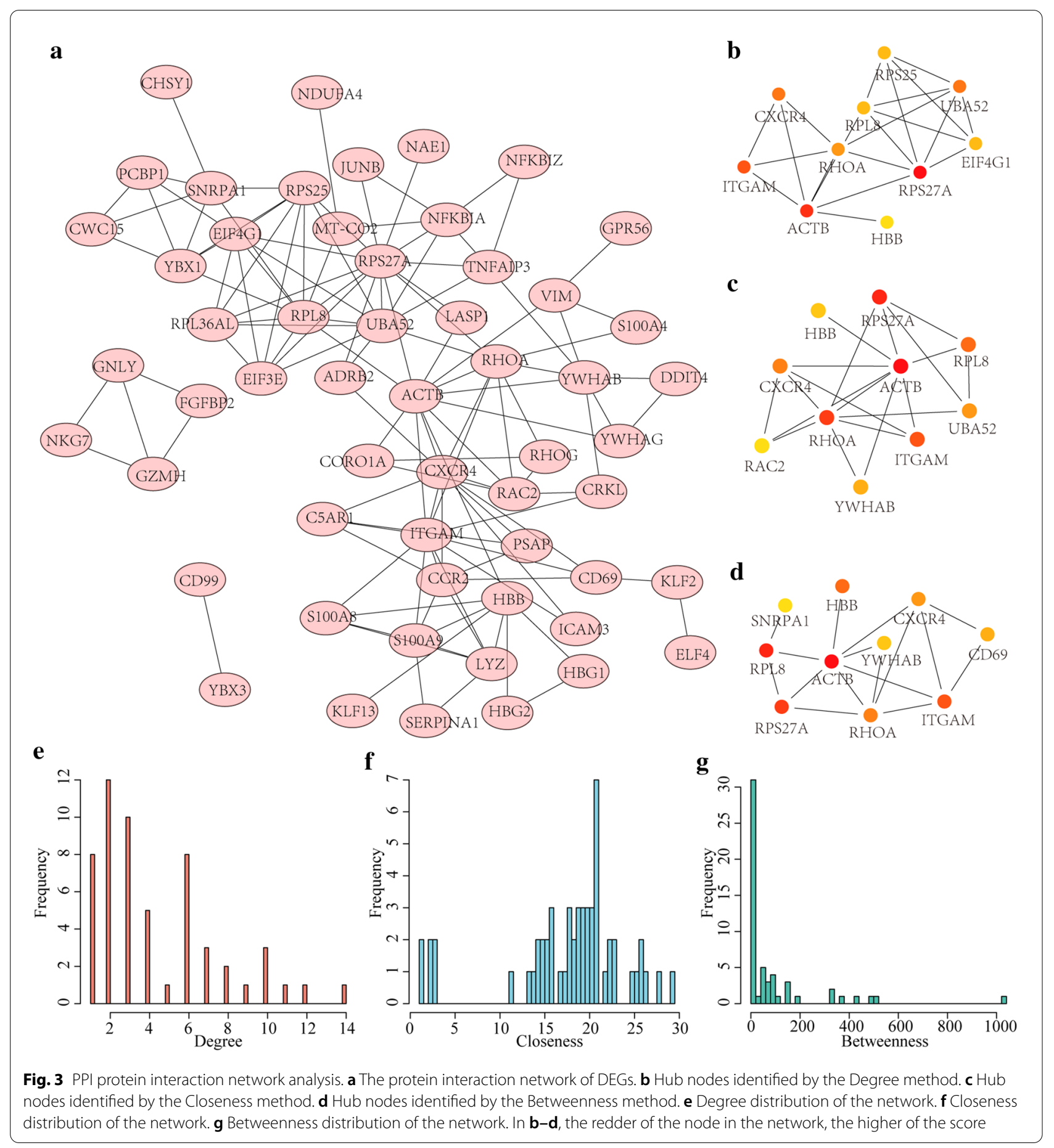

specificity of the model for MS were all $100 \%$, and the area under the receiver operating characteristic (ROC) curve was 1 (Fig. 4b, c). We merged the GSE21942 and GSE43591 datasets and applied the model to the merged dataset with an AUC of 0.96 (Fig. 4d). In the independent validation set GSE15245, 63 out of 65 samples were correctly classified with $96.92 \%$ classification accuracy, and the sensitivity and specificity of the model for MS were $100 \%$ and $85.71 \%$, respectively, and the area under the ROC curve (AUC) was 0.929 (Fig. 4e). In addition, in the GSE15245 data set, the model was applied to samples of different genders, and it was observed that the AUC of Male samples was 0.916 (Additional file 2: Figure S2A) and that of Female samples was 0.91 (Additional file 2: 

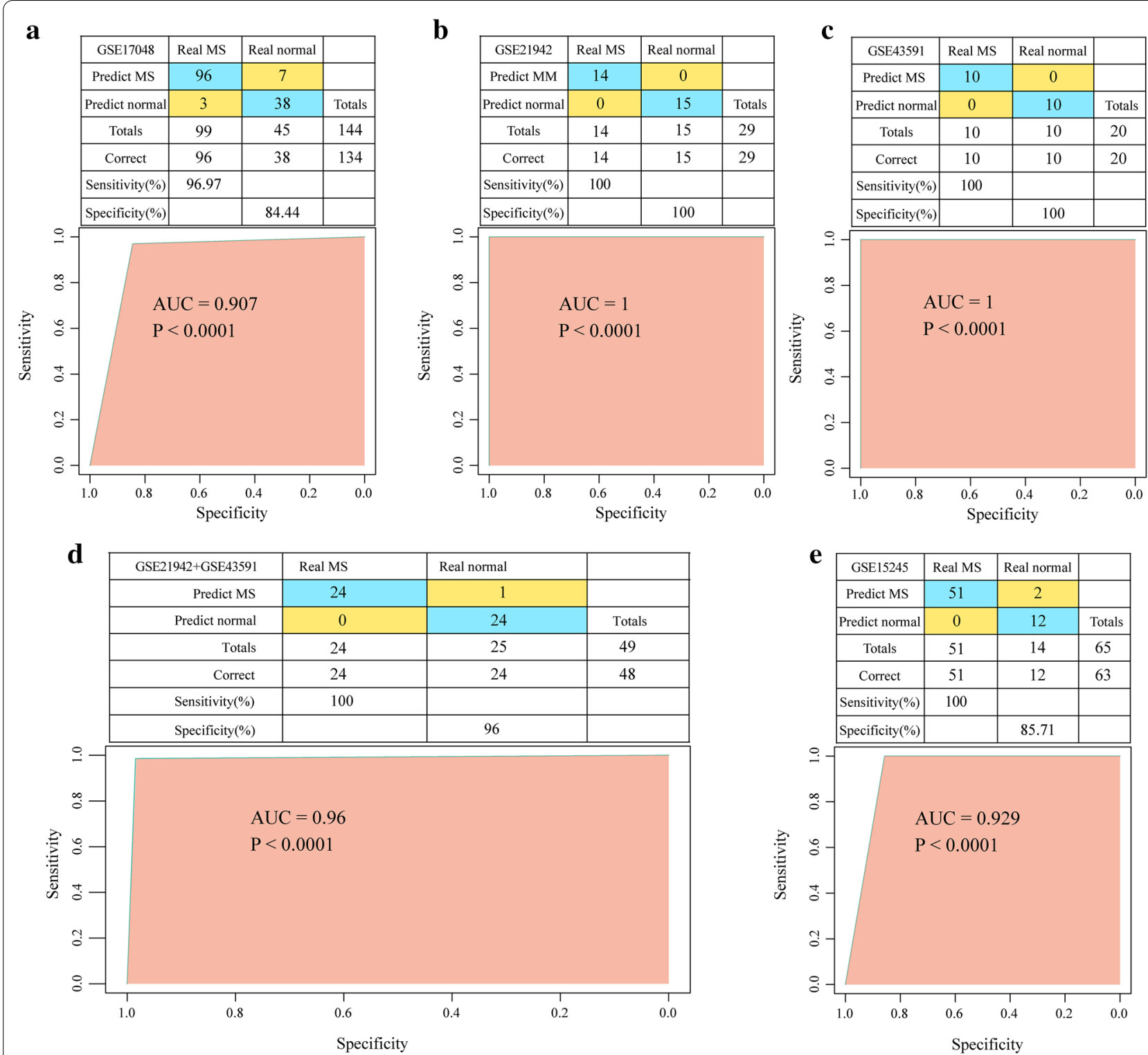

Fig. 4 Construction of diagnostic model and validation of model. a Classification results and ROC curves of samples by diagnostic model in training data set. $\mathbf{b}$ Classification results and ROC curves of samples by diagnostic model in GSE21942. c Classification results and ROC curves of samples by diagnostic model in GSE43591. d Classification results and ROC curves of samples by diagnostic model in GSE43591 + GSE21942. e Classification results and ROC curves of samples by diagnostic model in GSE15245

Figure S2B), indicating that the prediction performance was similar in samples of different genders. According to the age distribution of the sample, the AUC of the model in the $>30$ years sample was 0.9 (Additional file 2: Figure $\mathrm{S} 2 \mathrm{C}$ ) and the AUC of the model in the $<=30$ years sample was 0.88 (Additional file 2: Figure S2D), suggesting that the predictive performance of the model was similar across age groups. To further confirm the role of the seven genes, RT-qPCR was performed to detect the expressions of genes in the PBMC samples of MS patients. We found that in the PBMC samples of MS patients when compared with normal samples, CXCR4, RPL8 and RPS27A were obviously up-regulated, and UBA52 and RHOA were down-regulated (Fig. 5). Though the sample size may be relatively small, these results indicated that the diagnostic prediction model constructed in this study can effectively distinguish patients with MS from normal controls, and that the seven hub genes can be used as reliable biomarkers for MS diagnosis. 


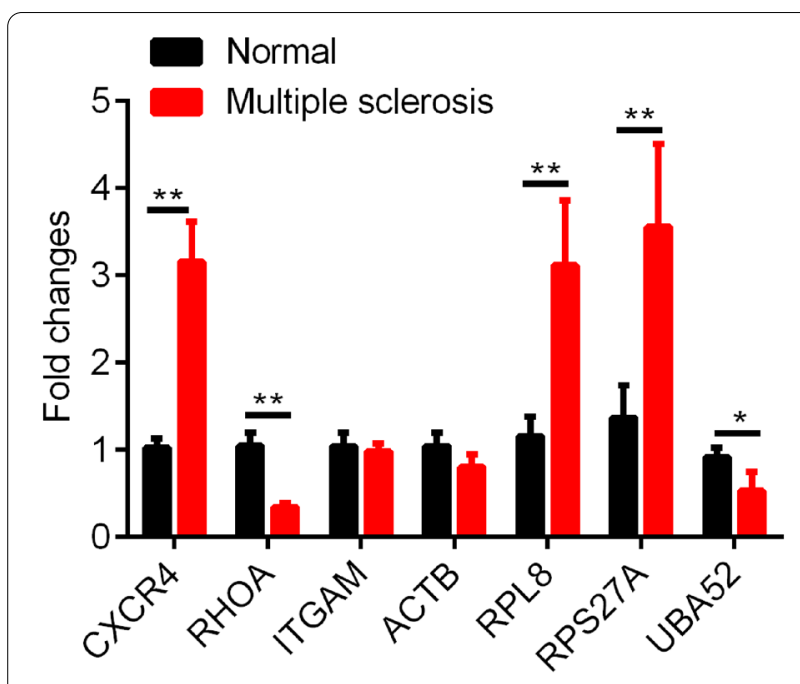

Fig. 5 Differential expression analysis of CXCR4, ITGAM, ACTB, RHOA RPS27A, UBA52, and RPL8 in MS patient PBMC using RT-qPCR assay

\section{Discussion}

As is a chronic and progressive autoimmune disease, multiple sclerosis (MS) is a leading cause of disability to young adults [20]. However, the pathogenesis and progression of MS remain unclear. In this study, gene expression profiles of peripheral blood samples were analyzed by bioinformatics based on multiple microarray datasets. The results of functional enrichment analysis showed that differentially expressed genes were associated with Toll-like receptor signaling pathway, pattern recognition receptor signaling pathway, innate immune response-activating signal transduction, leukocyte transendothelial migration, IL-17 signaling pathway and immune-related signaling pathways. In MS, abnormalities of immune systems involve leukocyte transendothelial migration, IL-17 signaling pathway, and abnormalities of innate immune response-activating signal transduction pathway, here, innate and adaptive immunity play an important role $[10,15]$. Moreover, immune dysregulation has been confirmed as an important mechanism in MS. It has been shown that leukocyte transendothelial migration is a driving factor in initiating an inflammatory immune response [28]. Above findings indicate that the pathogenesis of MS is multifactorial, complex, and is influenced by inflammation and external environment. CXCR4, ITGAM, ACTB, RHOA, RPS27A, UBA52, and RPL8 genes were identified as hub genes of the PPI network. Among them, CXCR4, ITGAM, ACTB, and RHOA are jointly involved in the Rap1 signaling pathway, leukocyte transendothelial migration, regulation of actin cytoskeleton processes; RPS27A, UBA52 and
RPL8 genes are implicated in the ribosomal pathway (Additional file 1: Figure S1B).

CXCR4 is expressed in a variety of tissues, including in lymph nodes, brain, liver, colon, kidney, testis, lung, pancreas, skin and placenta, and in different cell types such as stromal cells, osteoblasts, fibroblasts, dendritic cells and monocytes [29, 30]. Focal areas of myelin destruction observed in MS often occur on a background of inflammation dominated by T-lymphocytes, hematogenous macrophages, microglial activation, and the presence of few B-lymphocytes and plasma cells [31, 32]. In vitro studies have shown that microglial activation leads to upregulation of CXCR4 [33, 34].

ITGAM is a major non-human leukocyte antigen, and plays an important role in leukocyte activation, adhesion and migration through stimulated endothelium and in phagocytosis of complement-encapsulated granules and neutrophil apoptosis [35]. ITGAM is associated with the pathogenesis of systemic lupus erythematosus (SLE) [36], and an increasing number of studies have shown a genetic association between ITGAM and various autoimmune diseases [37-39]. RHOA is a ubiquitously expressed cytoplasmic protein that belongs to the small GTPase family. RhoA acts as a molecular switch and is activated in response to the binding of chemokines, cytokines and growth factors, moreover, as RhoA regulates the activation of cytoskeletal proteins and other factors through the mDia and ROCK signaling cascades, it is therefore regarded as a key regulator of innate and adaptive immunity [27]. In animal models of MS, absence of RhoA in $\mathrm{T}$ cells will reduce the number of mature $\mathrm{T}$ cells in the thymus and spleen, thereby significantly attenuating the incidence and severity of MS. RhoA is a central regulator of several prototypical $\mathrm{T}$ cell responses and a new potential therapeutic target for diseases such as MS [40]. ITGAM and RHOA showed a high degree and closeness in our PPI, therefore, we speculated that ITGAM and RHOA may be involved in MS through the regulation of T-cell activation.

ACTB encodes $\beta$-actin, an abundant cytoskeletal housekeeping protein. Sharp reduction of ACTB protein will change cell shape, migration, proliferation and gene expression, thereby impairing the development of the brain, heart and kidneys [41]. Pathogenic variants of ACTB are commonly associated with Baraitser-Winter prefrontal brain syndrome, resulting in severe, persistent dystonia, developmental delays and sensorineural hearing loss [42]. ACTB has long been considered an endogenous housekeeping gene and has been widely used as a reference gene/protein to reflect gene expression in tumors. Evidence increasingly demonstrated that ACTB is dysregulated in liver, melanoma, kidney, colorectal, gastric, pancreatic, esophageal, lung, breast, prostate, ovarian, 
leukemia and lymphoma. The aberrant expression and aggregation of ACTB and alterations in the cytoskeleton are associated with cancer aggressiveness and metastasis [43], suggesting that ACTB may be expressed at different levels in different environments and under different experimental conditions.

So far, RPS27A, UBA52, and RPL8 have not been reported in MS, and our study suggests that these genes are worthy of further study in MS.

Support vector machine (SVM) is a popular machine learning method widely used with biomedical data analysis. In this study, a diagnostic model of MS was developed based on SVM using the expression profiles of seven potential markers, and had an AUC of 0.907 in the training set, suggesting the accuracy of using these seven hub genes in classifying MS. We first validated the model in the dataset GSE21942 of the same platform with an AUC of 1 and an accuracy of 100\%, and further validated the model in the dataset GSE43591 of a different platform and obtained an AUC of 1. Such results indicated the applicability of our diagnostic model to data from different chip platforms. Finally, to examine the model's prediction of data from different sources, the dataset GSE15245 originated from blood samples was applied for verification and an AUC of 0.929 was obtained, which indicates the reproducibility of the model. Moreover, the 7-gene diagnostic model can also be used to classify patients based on blood samples, thus, our model may have broad application prospects in clinical practice.

Although we used bioinformatics techniques to identify potential candidate markers involved in MS occurrence from a large sample, the study also has several limitations. Firstly, the sample lacked clinical follow-up information, thus, we did not consider factors such as the presence of other health states of the patient in affecting the identification of diagnostic biomarkers from the samples. Secondly, the results obtained only by bioinformatics analysis will require further experimental validation. Third, the study does not include any clinically isolated syndrome (CIS) cases or patients with other neurological diseases that make up the differential diagnoses for MS. Lastly, the model only benefited for MS patients. Therefore, further genetic and experimental studies with larger sample sizes and experimental validation are needed.

\section{Conclusions}

In summary, in this study, we systematically analyzed the gene expression profiles of 248 blood samples, and constructed an aberrantly expressed gene signature involving a variety of important biological pathways for MS. Finally, we determined seven potential markers for MS based on protein interaction networks and developed a highly accurate diagnostic model, which is applicable to different microarray platforms and can be used in blood samples (mean AUC $=0.96$ ). The findings of this study provide targets and references for clinicians and bio-laboratory scientists.

\section{Methods}

Briefly, this study began with data collection and differential expression analysis, and multiple data integration analysis to identify key differential genes, followed by function enrichment analysis, protein interaction network construction, feature selection, and construction and validation of the classifier (Fig. 6).

\section{Data collection}

The NCBI GEO database was systematically searched to identify GEO datasets with microarray expression data relevant to MS. Only studies conducted with peripheral blood were included in our analysis. Data sets with $<10$ samples and studies without control samples were excluded. MS pathology types were PPMS/RRMS/ SPMS/CIS and samples derived from patients prior to drug treatment were included to subsequent analyses. Finally, four datasets (GSE21942, GSE43591, GSE15245, and GSE17048) were screened. GSE21942 and GSE43591 were downloaded from Affymetrix Human Genome U133 Plus 2.0 Array platform, GSE15245 and GSE17048 were respectively downloaded from Affymetrix Human Genome U133A 2.0 Array platform data and Illumina HumanHT-12 V3.0 expression beadchip. We obtained standardized expression profiling data of 84 control samples and 174 MS samples from the GEO database, and the sample information for each dataset was shown in Table 1. The GSE21942, GSE43591, and GSE15245 data sets are standardized by RMA, and the GSE17048 data sets are standardized by cubic spline. The probes were matched to genes, and those matched to multiple genes were removed. When multiple probes matched to one gene, the median of these probes were taken as the expression value for the gene. Here, we obtained four gene expression profiles.

\section{Integration of multiple data sets to identify differentially expressed genes}

The differentially expressed genes (DEGs) between normal samples and MS samples were screened by R software package limma [44] in GSE21942, GSE43591 and GSE17048. To include more genes with high differences, $\mathrm{FDR}<0.05$ and $\mid \log 2$ (Foldchange) $\mid>1$ was used as the thresholds to screen DEGs. The R package RobustRankAggreg [45] was used to integrate the DEGs screened from the three gene expression profiles, and genes with a score value less than 0.01 were considered as the DEGs. The distribution of fold change of DEGs in different data 


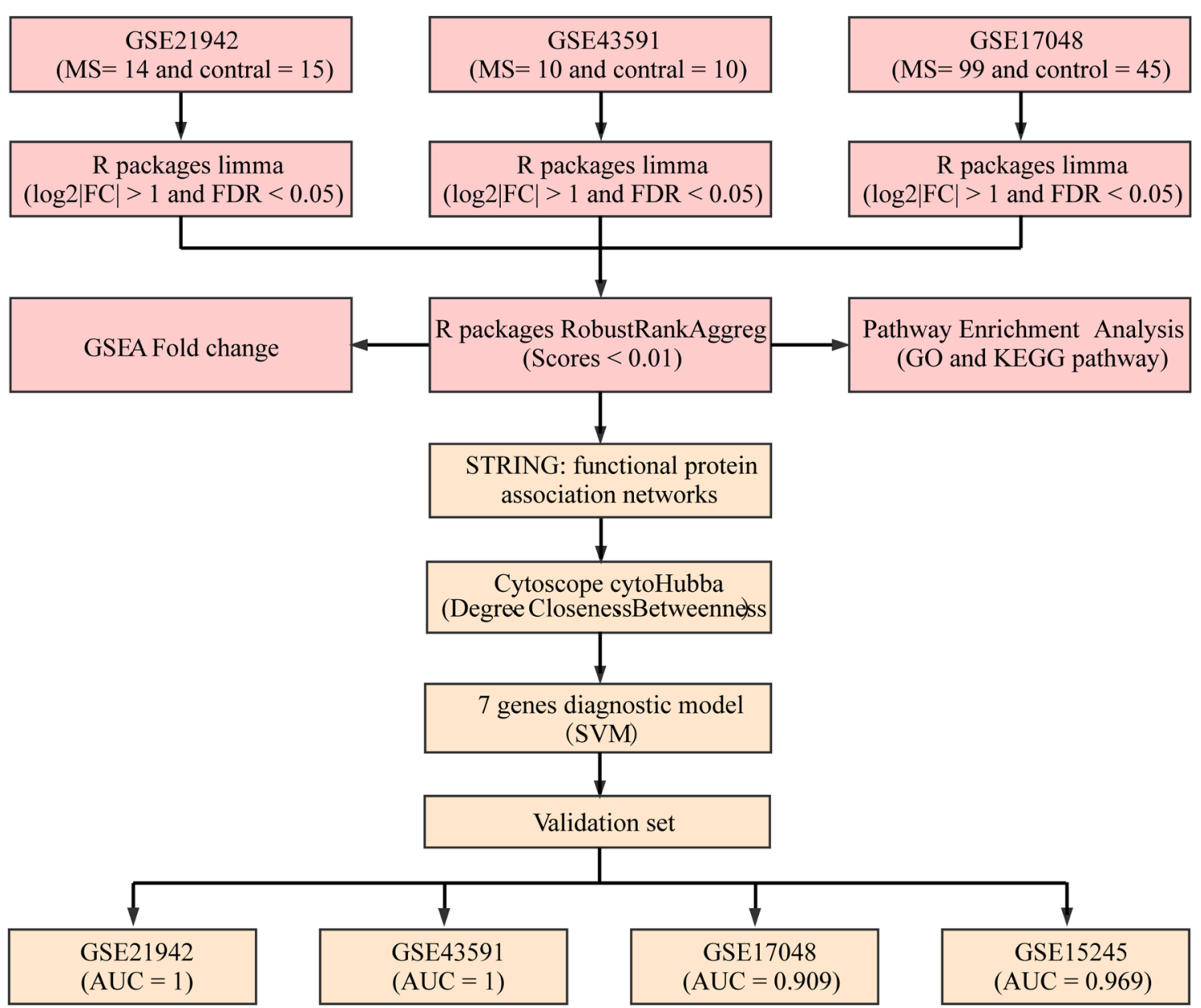

Fig. 6 The workflow of the study

Table 1 Studies and data included in this analysis

\begin{tabular}{|c|c|c|c|c|}
\hline \multirow[t]{2}{*}{ GEO accession } & \multicolumn{2}{|c|}{ Sample size } & \multirow[t]{2}{*}{ Sample source } & \multirow[t]{2}{*}{ Platform } \\
\hline & MS case & Control & & \\
\hline GSE21942 & 14 & 15 & Peripheral blood & GPL570 Affymetrix Human Genome U133 Plus 2.0 Array \\
\hline GSE43591 & 10 & 10 & Peripheral blood & GPL570 Affymetrix Human Genome U133 Plus 2.0 Array \\
\hline GSE15245 & 51 & 14 & Peripheral blood & GPL571 Affymetrix Human Genome U133A 2.0 Array \\
\hline GSE17048 & 99 & 45 & Peripheral blood & GPL6947 Illumina HumanHT-12 V3.0 expression beadchip \\
\hline Total & 174 & 84 & & \\
\hline
\end{tabular}

sets and the enrichment relationship of DEGs in different data sets were examined by performing GSEA analysis of DEGs using the rank order of fold change of genes in each data set as a background.

\section{Functional enrichment analyses}

Gene Ontology (GO) and Kyoto Encyclopedia of Genes and Genomes (KEGG) pathway enrichment analysis were performed using the clusterProfiler [46] for analyzing the gene relationship with DEGs. Subsequently, over-represented GO terms in biological processes and KEGG pathway were identified and visualized using the R package GOplot [47]. For both analyses, FDR $<0.05$ was considered to denote statistical significance. 
Protein interaction network construction and identification of key genes

The STRING Database (https://string-db.org/), which is an online platform for predicting gene interactions, is designed to collect, evaluate and integrate all public "protein-protein" interaction resources and to complement the results of computer predictions [48]. To analyze the interaction correlations of DEGs, we mapped DEGs to the STRING (version 11.0) database to obtain the interaction relationships among the genes. A combined score $>0.4$ served as the threshold to establish a PPI network, and then the topological properties of the network was visualized and analyzed by Cytoscape24 software (version 3.7.1) [49]. In addition, the plug-in cytoHubba25 [50] in Cytoscape software was used to calculate the network's degree, closeness and betweenness for identifying key genes from the PPI network.

\section{Construction of MS diagnostic prediction model and assessment of predictive performance of the model}

Support Vector Machine (SVM) is a supervised learning model for machine learning algorithms and can be used to analyze data and identify gene expression patterns. The SVM could construct a hyperplane in high or infinite dimensional space for classification and regression. Specifically, based on a set of training samples with each marker belonging to one of two categories, a SVM training algorithm builds a model that assigns new instances to one or multiple categories, making it a non-probabilistic binary linear classification. Here, according to the expression profiles of key genes, we constructed a diagnostic prediction model based on SVM classification (51). We used GSE17048 as a training dataset, and GSE21942 and GSE43591 as validation sets. Additionally, GSE15245 served as an independent set of external validation sets to validate the prediction performance of the model. The model was constructed in the training dataset and its classification capability was validated using a tenfold cross-validation method. Samples from the validation dataset were predicted using the built model. The predictive ability of the model was reflected by the area under the ROC curve (AUC), and the sensitivity and specificity of the model to predict MS patients were analyzed.

\section{RT-qPCR}

TRIzol (Thermo, 15,596,026) were applied to extract total RNA from peripheral blood mononuclear cell (PBMC), included 4 MS patients and 5 control samples. The synthesis of total RNA into cDNA was conducted according to the instructions of the reverse transcription kit (Thermo, BTK1622). Amplification was performed by conducting real-time polymerase chain reaction in an ABI 7500 real-time PCR instrument with an one-step qRT-PCR Kit (FP303, Tiangen Biochemical Technology (Beijing) Co., Ltd.). The primers were as follows: CXCR4 forward, CTTGACACTGGATAT ACACTTCAG and reverse, AACAGGGTTCCTTCA TGGAG; ITGAM forward, CAATATCAGGTCAGC AACCTG and reverse, ATGACAGTCTGGTTCAGCC; ACTB forward, GAAGATCAAGATCATTGCTCCTC and reverse, ATCCACATCTGCTGGAAGG; RHOA forward, AGTTCCCAGAGGTGTATGTG and reverse, CCAACTCTACCTGCTTTCCA; GAPDH forward, TCAAGATCATCAGCAATGCC and reverse, CGA TACCAAAGTTGTCATGGA; GAPDH was an internal control. $2^{-\triangle \triangle \mathrm{Ct}}$ was used to calculate the relative expressions. The $\mathrm{T}$ test was used to analyze the differences in gene expression between the two groups.

\section{Abbreviations \\ MS: Multiple sclerosis; PPI: Protein-protein interaction; SVM: Support vector machine approach; EBV: Epstein-Barr virus; CNS: Central nervous system; BBB: Blood-brain barrier; DEGs: Differentially expressed genes; GO: Gene Ontology; KEGG: Kyoto Encyclopedia of Genes and Genomes.}

\section{Supplementary Information}

The online version contains supplementary material available at https://doi. org/10.1186/s40659-021-00334-6.

Additional file 1: Figure S1. ROC analysis and KEGG analysis. A AUC of gene combinations identified under different thresholds in the training set. B The KEGG Pathway where CXCR4, ITGAM, ACTB, RHOA, RPS27A, UBA52 and RPL8 genes participate together.

Additional file 2: Figure S2. Classification performance of MS diagnostic model in genders and ages in GSE15245 dataset. A The classification result and ROC curve of the MS sample of the diagnostic model in the Male sample; B The classification result and ROC curve of the MS sample of the diagnostic model in the Female sample; C The classification result and ROC curve of the MS sample of the diagnostic model in the Age $>30$ samples; $\mathbf{D}$ The classification result and ROC curve of the MS sample of the diagnostic model in the $v$ samples.

Acknowledgements

Not applicable.

\section{Authors' contributions}

Conception and design of the research: XC, MD. Acquisition of data: $\mathrm{HH}, \mathrm{HQ}$. Analysis and interpretation of data: HF. Statistical analysis: TZ. Drafting the manuscript: XC. Revision of manuscript for important intellectual content: MD. All authors read and approved the final manuscript.

Funding

None.

Availability of data and materials

The datasets used and/or analyzed during the current study are available from the corresponding author on reasonable request. 


\section{Declarations}

Ethics approval and consent to participate

The experiments were approved by the Ethics Committee of the Second Hospital of Hebei Medical University, and the informed consent was signed by all the participants.

\section{Consent for publication}

Not applicable.

\section{Competing interests}

The authors declare that they have no competing interests.

Received: 30 September 2020 Accepted: 8 March 2021

Published online: 01 April 2021

\section{References}

1. Yamout BI, Alroughani R. Multiple Sclerosis. Semin Neurol. 2018;38(2):212-25.

2. Orton SM, Herrera BM, Yee IM, Valdar W, Ramagopalan SV, Sadovnick AD, et al. Sex ratio of multiple sclerosis in Canada: a longitudinal study. Lancet Neurol. 2006;5(11):932-6.

3. Weinshenker BG. Epidemiology of multiple sclerosis. Neurol Clin. 1996;14(2):291-308

4. Mayr WT, Pittock SJ, McClelland RL, Jorgensen NW, Noseworthy JH, Rodriguez M. Incidence and prevalence of multiple sclerosis in Olmsted County, Minnesota, 1985-2000. Neurology. 2003;61(10):1373-7.

5. Tullman MJ. Overview of the epidemiology, diagnosis, and disease progression associated with multiple sclerosis. Am J Manag Care. 2013;19(2 Suppl):S15-20.

6. Ascherio A. Environmental factors in multiple sclerosis. Expert Rev Neurother. 2013;13(12 Suppl):3-9.

7. Ascherio A, Munger KL. Environmental risk factors for multiple sclerosis. Part II: Noninfectious factors Ann Neurol. 2007;61(6):504-13.

8. Wingerchuk DM. Smoking: effects on multiple sclerosis susceptibility and disease progression. Ther Adv Neurol Disord. 2012;5(1):13-22.

9. Farez MF, Fiol MP, Gaitan MI, Quintana FJ, Correale J. Sodium intake is associated with increased disease activity in multiple sclerosis. J Neurol Neurosurg Psychiatry. 2015;86(1):26-31.

10. Selter RC, Hemmer B. Update on immunopathogenesis and immunotherapy in multiple sclerosis. Immunotargets Ther. 2013;2:21-30.

11. Hafler DA, Slavik JM, Anderson DE, O'Connor KC, De Jager P, Baecher-Allan C. Multiple sclerosis. Immunol Rev. 2005;204:208-31.

12. Dette C, Boettcher SW. Surface science: Catalytic hotspots get noisy. Nature. 2017;549(7670):34-5.

13. Jelcic I, Al Nimer F, Wang J, Lentsch V, Planas R, Jelcic I, et al. Memory $B$ cells activate brain-homing, autoreactive CD4(+) T cells in multiple sclerosis. Cell. 2018;175(1):85-100.e23.

14. Inglese M. Multiple sclerosis: new insights and trends. AJNR Am J Neuroradiol. 2006;27(5):954-7.

15. O'Connor KC, Bar-Or A, Hafler DA. The neuroimmunology of multiple sclerosis: possible roles of T and B lymphocytes in immunopathogenesis. J Clin Immunol. 2001:21(2):81-92.

16. Berkovich R. Treatment of acute relapses in multiple sclerosis. Neurotherapeutics. 2013;10(1):97-105.

17. Ascherio A, Munger KL. Environmental risk factors for multiple sclerosis. Part I: the role of infection. Ann Neurol. 2007;61(4):288-99.

18. International Multiple Sclerosis Genetics C, Wellcome Trust Case Control C, Sawcer S, Hellenthal G, Pirinen M, Spencer CC, et al. Genetic risk and a primary role for cell-mediated immune mechanisms in multiple sclerosis. Nature. 2011:476(7359):214-9.

19. Hemmer B, Kerschensteiner M, Korn T. Role of the innate and adaptive immune responses in the course of multiple sclerosis. Lancet Neurol. 2015:14(4):406-19.

20. Chung SE, Cheong HK, Park JH, Kim HJ. Burden of disease of multiple sclerosis in Korea. Epidemiol Health. 2012:34:e2012008.
21. Nickles D, Chen HP, Li MM, Khankhanian P, Madireddy L, Caillier SJ, et al. Blood RNA profiling in a large cohort of multiple sclerosis patients and healthy controls. Hum Mol Genet. 2013:22(20):4194-205.

22. Bomprezzi R, Ringner M, Kim S, Bittner ML, Khan J, Chen Y, et al. Gene expression profile in multiple sclerosis patients and healthy controls: identifying pathways relevant to disease. Hum Mol Genet. 2003;12(17):2191-9.

23. Creanza TM, Liguori M, Liuni S, Nuzziello N, Ancona N. Meta-analysis of differential connectivity in gene co-expression networks in multiple sclerosis. Int J Mol Sci. 2016;17:6.

24. Zepp JA, Liu C, Qian W, Wu L, Gulen MF, Kang Z, et al. Cutting edge: TNF receptor-associated factor 4 restricts IL-17-mediated pathology and signaling processes. J Immunol. 2012;189(1):33-7.

25. Lou J, Gasche Y, Zheng L, Giroud C, Morel P, Clements J, et al. Interferonbeta inhibits activated leukocyte migration through human brain microvascular endothelial cell monolayer. Lab Invest. 1999;79(8):1015-25.

26. Pozzobon T, Goldoni G, Viola A, Molon B. CXCR4 signaling in health and disease. Immunol Lett. 2016:177:6-15.

27. Bros M, Haas K, Moll L, Grabbe S. RhoA as a key regulator of innate and adaptive immunity. Cells. 2019:8:7.

28. Getter T, Margalit R, Kahremany S, Levy L, Blum E, Khazanov N, et al. Novel inhibitors of leukocyte transendothelial migration. Bioorg Chem. 2019;92:103250.

29. Garcia-Cuesta EM, Santiago CA, Vallejo-Diaz J, Juarranz Y, Rodriguez-Frade JM, Mellado M. The role of the CXCL12/CXCR4/ACKR3 axis in autoimmune diseases. Front Endocrinol (Lausanne). 2019;10:585.

30. Hendrickx DAE, van Scheppingen J, van der Poel M, Bossers K, Schuurman KG, van Eden CG, et al. Gene expression profiling of multiple sclerosis pathology identifies early patterns of demyelination surrounding chronic active lesions. Front Immunol. 2017;8:1810.

31. Noseworthy JH, Lucchinetti C, Rodriguez M, Weinshenker BG. Multiple sclerosis. N Engl J Med. 2000;343(13):938-52.

32. BrückW. The pathology of multiple sclerosis is the result of focal inflammatory demyelination with axonal damage. J Neurol. 2005;252(Suppl 5):v3-9.

33. Flynn G, Maru S, Loughlin J, Romero IA, Male D. Regulation of chemokine receptor expression in human microglia and astrocytes. J Neuroimmunol. 2003;136(1-2):84-93.

34. Ohtani Y, Minami M, Kawaguchi N, Nishiyori A, Yamamoto J, Takami S, et al. Expression of stromal cell-derived factor-1 and CXCR4 chemokine receptor mRNAs in cultured rat glial and neuronal cells. Neurosci Lett. 1998:249(2-3):163-6.

35. Fagerholm SC, Varis M, Stefanidakis M, Hilden TJ, Gahmberg CG. alphaChain phosphorylation of the human leukocyte CD1 1b/CD18 (Mac-1) integrin is pivotal for integrin activation to bind ICAMs and leukocyte extravasation. Blood. 2006;108(10):3379-86.

36. Fan Y, Li LH, Pan HF, Tao JH, Sun ZQ, Ye DQ. Association of ITGAM polymorphism with systemic lupus erythematosus: a meta-analysis. J Eur Acad Dermatol Venereol. 2011:25(3):271-5.

37. Anaya JM, Kim-Howard X, Prahalad S, Chernavsky A, Canas C, RojasVillarraga $A$, et al. Evaluation of genetic association between an ITGAM non-synonymous SNP (rs1143679) and multiple autoimmune diseases. Autoimmun Rev 2012:11(4):276-80.

38. Warchol T, Lianeri M, Lacki JK, Olesinska M, Jagodzinski PP. ITGAM Arg77His is associated with disease susceptibility, arthritis, and renal symptoms in systemic lupus erythematosus patients from a sample of the Polish population. DNA Cell Biol. 2011;30(1):33-8.

39. Lee $Y H$, Bae SC. Association between the functional ITGAM rs 1143679 $\mathrm{G} / \mathrm{A}$ polymorphism and systemic lupus erythematosus/lupus nephritis or rheumatoid arthritis: an update meta-analysis. Rheumatol Int. 2015;35(5):815-23.

40. Manresa-Arraut A, Johansen FF, Brakebusch C, Issazadeh-Navikas S, Hasseldam H. RhoA Drives T-cell activation and encephalitogenic potential in an animal model of multiple sclerosis. Front Immunol. 2018;9:1235.

41. Cuvertino S, Stuart HM, Chandler KE, Roberts NA, Armstrong R, Bernardini $\mathrm{L}$, et al. ACTB loss-of-function mutations result in a pleiotropic developmental disorder. Am J Hum Genet. 2017;101(6):1021-33.

42. Conboy E, Vairo F, Waggoner D, Ober C, Das S, Dhamija R, et al. Pathogenic Variant in ACTB, p.Arg183Trp, causes juvenile-onset dystonia, hearing loss, and developmental delay without midline malformation. Case Rep Genet. 2017;2017:9184265. 
43. Guo C, Liu S, Wang J, Sun MZ, Greenaway FT. ACTB in cancer. Clin Chim Acta. 2013;417:39-44.

44. Ritchie ME, Phipson B, Wu D, Hu Y, Law CW, Shi W, et al. limma powers differential expression analyses for RNA-sequencing and microarray studies. Nucleic Acids Res. 2015;43(7):e47.

45. Kolde R, Laur S, Adler P, Vilo J. Robust rank aggregation for gene list integration and meta-analysis. Bioinformatics. 2012;28(4):573-80.

46. Yu G, Wang LG, Han Y, He QY. clusterProfiler: an R package for comparing biological themes among gene clusters. OMICS. 2012:16(5):284-7.

47. Walter W, Sanchez-Cabo F, Ricote M. GOplot: an R package for visually combining expression data with functional analysis. Bioinformatics. 2015;31(17):2912-4.

48. Szklarczyk D, Franceschini A, Wyder S, Forslund K, Heller D, Huerta-Cepas J, et al. STRING v10: protein-protein interaction networks, integrated over the tree of life. Nucleic Acids Res. 2015;43(Database issue):D447-52.
49. Shannon P, Markiel A, Ozier O, Baliga NS, Wang JT, Ramage D, et al. Cytoscape: a software environment for integrated models of biomolecular interaction networks. Genome Res. 2003;13(11):2498-504.

50. Chin $\mathrm{CH}$, Chen SH, Wu HH, Ho CW, Ko MT, Lin CY. cytoHubba: identifying hub objects and sub-networks from complex interactome. BMC Syst Biol. 2014;8(Suppl 4):S11.

51. Sanz H, Valim C, Vegas E, Oller JM, Reverter F. SVM-RFE: selection and visualization of the most relevant features through non-linear kernels. BMC Bioinformatics. 2018;19(1):432.

\section{Publisher's Note}

Springer Nature remains neutral with regard to jurisdictional claims in published maps and institutional affiliations.
Ready to submit your research? Choose BMC and benefit from:

- fast, convenient online submission

- thorough peer review by experienced researchers in your field

- rapid publication on acceptance

- support for research data, including large and complex data types

- gold Open Access which fosters wider collaboration and increased citations

- maximum visibility for your research: over $100 \mathrm{M}$ website views per year

At BMC, research is always in progress.

Learn more biomedcentral.com/submissions 